\title{
Comparative Evaluation of Supply Chain Management Strategies: A Simulation Approach
}

\author{
Felix T. S. Chan
}

Department of Industrial and Manufacturing Systems Engineering

The University of Hong Kong

Pokfulam Road, Hong Kong

E-mail: ftschan@hkucc.hku.hk

\begin{abstract}
Supply chain management has become one of the most important sources of competitive advantage in different industries. For years, researchers have investigated the various processes within supply chain. There has been an increasing attention placed on performance, design and analysis of supply chain. However, the supply chain is a complex model so it is difficult to analyze the performance of a supply chain philosophically. To evaluate the control mechanism for a supply chain, simulation is one of the effective methods. There are five common supply chain models built up in this paper. From the simulation results, various performance measures such as transportation cost, resources utilization, inventory level, and order cycle time can be calculated.
\end{abstract}

\section{Introduction}

Supply chain management is now being watched with interest in order to manage the network of companies complexity efficiently. In a supply chain, however, a slight change of management strategy in one company largely affects the whole efficiency of the supply chain. Furthermore, there are many uncertain factors, so it is difficult to analyze performance of a supply chain philosophically. In this connection, a simulation toolSIMPROCESS is introduced in this paper. By using simulation techniques, the performance of a supply chain model can be evahuated. In addition, Analytic Hierarchy Process (AHP) method was used to define which model is the optimal. AHP method was introduced by Satty (1990), which can be used to measure and quantify both quantitative and qualitative attributes. Also, it can structure a complex, multi-attribute, muti-objective hierarchically, and make easily pairwise comparisons between elements.

In this paper, there are mainly 4 echelons in supply chain model namely Suppliers, Manufacturers, Retailers, and Customers. The number in each echelon was fixed. Also, in each model category, the path and quantity of order distribution was different. The purpose of this paper is to determine which supply chain model can achieve the optimal performance in the four measurements inventory level, order lead time, resources utilization, and transportation cost. Five models were developed, and each of them has different characteristics. However, they could be grouped into three main categories- Interorganization supply chain, Network supply chain, and Regional clustering supply chain.

\section{General Model Pattern}

Supply chain is a chain of units that transfers demand data from customers to suppliers, and converts the materials received from the supplier into products and services which will be delivered to customers. In this paper, there are mainly four echelons in supply chain model-Suppliers, Manufacturers, Retailers, and Customers. There are three suppliers (Supplier A, Supplier B, and Supplier C), three Manufacturers (Manufacture A, Manufacture B, and Manufacture C), two Retailers (Retailer A, and Retailer B), and three Customers (Customer A, Customer B, and Customer C).

\subsection{General Assumptions}

1. Pull-based supply chain Production is demand driven that coordinated with actual customer demand rather than a forecast.

2. Excluded communication time Communication times between echelon to echelon, layer to layer, are neglected.

3. Order quantity generated by customers Order quantity generated by customers was governed by normal distribution with a mean value of 1500 units and standard deviation of 450 units, and the order is generated periodically.

4. Materials provided by Suppliers as requested by manufacturers

If the order quantity is less or equal b 800 in supplier $\mathrm{A}$, it could provide the material at once. However, if the order quantity is over the limitation, different models possess different approaches. For example, in Model 1,once the order quantity is over limitation, the order will be delayed for one day to allow for the supplier preparation. Consequently, the supplier will provide a fix economic batch of 2000 units in another day. In Model 2, if the order quantity were over the limit, then the whole order would send to Supplier B where larger order quantity could be provided. In this paper, the limitation of Supplier B is 1200 where as there is no limitation in Supplier C.

5. Transportation costs concerned in supplier and manufacturer echelon 
(Transportation time of using truck A, B, or C is the same)

- Supplier A and Manufacturer A use Truck A (transportation cost is $\$ 2,000$ / entity).

- Supplier B and Manufacturer B use Truck B (transportation cost is $\$ 3,000$ / entity).

- Supplier C and Manufacturer C use Truck C (transportation cost is $\$ 4,000$ / entity)

6. Simulation Time

In the experiment, the simulation period is one year.

2.2 Model Description

In this paper, there are totally five developed models. In fact, these five models could be grouped into three main categories. There is inter-organizational supply chain (Model 1); network supply chain (Models 2-4), and regional clustering supply chain (Model 5).

\subsubsection{Inter-organizational supply chain}

In the supply chain model, Harland (1998) defined a term called 'dyadic', meaning that one buyer-one seller. In fact, most of the supply chain research papers had been emphasized on this model.

\section{Characteristic of Model I}

Figure 1 shows the flow of Model 1. The characteristics of Model 1 are stated as follows:

1. Customer Echelon to Retailer Echelon - Customer A sends orders to Retailer A only. - Customers B and C send orders to Retailer B only.

2. Retailer Echelon to Manufacturer Echelon

- Retailer A sends order to Manufacturer A.

- Retailer B sends order to both Manufacturers B and $\mathrm{C}$.

3. Manufacture Echelon to Supplier Echelon

If inventory were not enough from the Manufacturers, they would send order to Supplier, otherwise, the goods from the stock of the manufacturer would be sent back to the Retailers at once.

- Manufacturer A sends order to Supplier A

- Manufacturer B sends order to Supplier B

- Manufacturer C sends order to Supplier C

If the order quantity was over the supplier's supply limit, the process in the supplier echelon would delay by 1 day. For example, the limitation of the supplier A is 800 units, which is mentioned in the previous assumptions. If the order quantity is more than 800 units from the Manufacturer A, the supplier could not supply immediately, but the material would be delayed by one day with a fix quantity of 2000 units.

\subsubsection{Network supply chain}

This type of supply chain models (Figure 2) had attracted very little attention until the late 1980s (Jarillo, 1998). However, it was not until the early 1990s that some research which was carried out in this area; in general, this tends to give a more real-world feel to the research but at the expense of adding greater complexity to the research activity. In this connection, Models 24 were built up. The concepts of communication between supplier layers, also between manufacturer layers, were introduced. These kinds of models are more realistic. If the supply chain model is lack of co-ordination and linkage between the various parties in the chain, this is definitely an inefficiencies of supply chain model.

\section{Characteristics of Model 2}

Figure 2 shows the flow of Model 2. The characteristics of model 2 are stated as follows.

\section{Customer Echelon to Retailer Echelon}

- Customer A $50 \%$ probability sends order to Retailer A and $50 \%$ to Retailer B

- Customer B 50\% probability sends order to Retailer A and $50 \%$ to Retailer B

- Customer C sends all order to Retailer B.

2. Retailer Echelon to Manufacturer Echelon

As the consideration of the transportation cost, most of the companies would prefer the lower cost. Similar as in this model, all the three manufacturers prefer the transportation option which offers the lowest cost. Therefore, all these manufacturers would prefer Truck A, second preference is Truck B, and the last choice is Truck $C$.

In Model 2, Retailer A and Retailer B would send the order to the Manufacturer A first (Truck $A$ is used in Manufacturer A). Then the Manufacturer A would check the stock whether the inventory is sufficient for the order. If the inventory was sufficient, then the goods in stock would be sent back to the retailers at once. However, if the inventory was not sufficient, the manufacturer A would send the entire order to the Manufacturer $B$. Similar to Manufacturer $A$, the same process will be repeated for Manufacturers B and C. As a result, if Manufacturer $C$ found insufficient stock, then the order would send to the suppliers. All the order would send to the Supplier A first as truck A was used in the Supplier A.

3. Manufacturer Echelon to Supplier Echelon

From the above, if the stock of Manufacturer $\mathrm{C}$ was not sufficient, all the orders would send to the Supplier A first. If the order quantity was smaller or equal to 800 which is the Supplier A supplied limitation, then the fixed quantity of material (Quantity 2000 units which mentioned in the assumptions) would send back to the Manufacturer A immediately. Say the order quantity is 800 , then Manufacturer A would get the material 2000 units. For the rest of the material 1200 would put it in the stock. Otherwise, the order would be rejected from the supplier A to the Supplier B. Once the Supplier $B$ gets the order, it would check it out whether the 
order quantity is over limited (i.e. the limitation of the supplier B is 1200 .) If the order is under or equal to Supplier B's limitation, then the fixed quantity material, i.e. 2000 units, would be sent to the Manufacturer B. Otherwise, the order would send to the Supplier C, as there is no limitation in Supplier $\mathrm{C}$, so that the fixed batch would be provided.

\section{Characteristic of Model 3}

Model 3 basically is the modification of the Model 2. In the result of the Model 2, the resources utilization was quite low which is under $20 \%$. Also the major problem in Model 2 is high level of inventory (Table 1). This can be explained as the problem is happened in the manufacturer echelon. If the stock of the Manufacturer A was 500 , however, the order quantity was 600 . The Manufacturer A would reject the order to the Manufacturer B. In fact, it would keep the high inventory and lower resources utilization in the manufacturers. Therefore, a more realistic model, namely Model 3 was built up. The characteristics of Model 3 are stated as follows.

1. Customer Echelon to Retailer Echelon As the major problem is not happened in this echelon, therefore the route would be same as Model 2.

2. Retailer Echelon to Manufacture Echelon Retailers A and B send orders to Manufacture A as the cheapest transportation cost.

The operation logic is a bit different from the Model 2. In Model 2 the major problem is that the manufacturers' order would always be rejected, thus causing a very low resources utilization and high inventory level. In order to solve this problem, the available amount of the inventory in Manufacturer would be directly sent back to the Retailers first. For example, if the inventory in Manufacturer $A$ is 500 , and the order quantity is 600 . Manufacturer $A$ would send 500 unit goods directly to the retailers first. And the remaining order of 100 units would send to the Manufacturer $B$ and so on. In this connection, the problem of resources utilization and the inventory level could be ixpproved.

3. Manufacturer Echelon to Supplier Echelon

After the inventories of all the three manufacturers have been used up, the unfilled amount of order would be sent to suppliers. This unfulfilled amount would be distributed equally to the suppliers. For example, if the unfulfilled amount is 300 , then 100 would send to Supplier A, 100 would send to Supplier B and 100 would send to Supplier C. And each of them would directly send back to Manufacturer A, Manufacturer B, and Manufacturer $C$ respectively. If the left amount is more than a supplier's limit, the order would send to the next supplier and so on.

\section{Characteristic of Model4}

In Model 3, it can be observed that the resources utilization and inventory level had been improved as compared with Model 2 (Table 1). In order to verify whether Model 3 is a optimal one. Model 4 was built up. The objective of this model is to modify the Model 3 which is using another approach in the supplier echelon. The main different between Model 3 and Model 4 is that the unfulfilled order quantity would not equally distributed to the Suppliers. Rather, it would send the order to the Supplier A as the first preference as the transportation cost in Supplier $A$ is the cheapest. If the unfulfilled order was smaller or equal to 800 which is Supplier A's limited, then the Supplier A would send back the fix economic quantity of 2000 units to the Manufacturer A. However, if the unfulfilled order was bigger than 800 , then Supplier A would supply 800 units to the Manufacturer A first, the remaining order would send to the Supplier B. If the remaining order was less than 1200 which is under the limitation of the Supplier B, then the Supplier B would supply the material directly to the Manufacturer $B$ at once and so on.

\subsubsection{Regional clustering supply chain}

This last area involves taking the network supply chains' logic a stage further and addressing their impact on particular regions where a cluster of firms exist (Hall and Andriani, 1998). A supply chan here does not have to be linear; indeed it can take a very complex form such as many regional industrial systems (Nassimbeni, 1998), industry-based supplier associations or industrial network groups (Esain and Hines, 1997). As these regional clusters are very dynamic in nature and typically involve many dozens of firms, they are very difficult to study (fig. 5). For this model, it may be more realistic and complex as compared with the previous models. As this is the latest model developed, this is a majorresearch opportunity as it is the level that comes closest to the real world. Therefore, Model 5 was built up.

\section{Characteristics of Model 5}

Figure 3 presents the flow of information and material in model 5 . The main different from the last few models is that there is no stock in the manufacturers. The order would be directly sent to the suppliers and the suppliers would provide the material directly. The characteristics of model 5 are stated as below:

1. Customer Echelon to Retailer Echelon

Customers A, B, and C send orders to both Retailers $A$ and $B$ based on some probabilities functions as stated below:

- Customer A sends $50 \%$ order to Retailer A and $50 \%$ to Retailer B.

- Customer B sends $50 \%$ order to Retailer A and $50 \%$ to Retailer B.

- Customer C sends $50 \%$ order to Retailer A and $50 \%$ to Retailer B. 
2. Retailer Echelon to Manufacture Echelon

- Retailer A sends $331 / 3 \%$ order to Manufacturer A, $331 / 3 \%$ order to Manufacturer B, $331 / 3 \%$ order to Manufacturer $\mathrm{C}$.

- Retailer B sends $331 / 3 \%$ order to Manufacturer A, $331 / 3 \%$ order to Manufacturer B, $331 / 3 \%$ order to Manufacturer C.

3. Manufacturer Echelon to Supplier Echelon

Manufacturer would split out the order to the suppliers equally. For example, if the order quantity is 1500 in Manufacturer $A$, then the order wo uld be divided into 3 parts which is equally distributed. Consequently, Manufacturer A ill receive 500 units from each supplier.

3 Results and Discussions

Four parameters are employed to measure the performance of the supply chain models. The results are shown in Table 1.

\section{(i) Average order lead time}

Model 2 out performs all the other models. Model 2 is the network supply chain model. In fact, because of the 'co-managed inventory', some orders could be quickly fulfilled and hence provided quick response to the customers.

(ii) Resources utilization

Model 5 is the best one. Model 5 is the regional clustering supply chain model. The manufacturer would split out the order to the suppliers, so that the resources utilization keeps high in the Model 5.

(iii) Inventory level

The regional clustering supply chain model is the best. As the just-in-time deliveries by manufacturers and suppliers are assumed, so that no stock is required in the manufacturers.

(iv) Transportation cost

The traditional model i.e. Model 1, Interorganizational supply chain model is the best. It is obvious that the order just sent to the one Manufacturer and vice versa. Therefore, the transportation cost is the lowest.

\subsection{Overall Performance Using AHP Method}

In the AHP by Satty (1990), the hierarchical pairwise comparison is employed to induce the relative weights of alternatives through pairwise comparison. Decisionmakers choose a value from a scale to express the relative significance of one alternative over another based on a Saaty's scale. All of the pairwise comparison values can be summarized in a comparison matrix, from which the relative weights of all the alternatives can be extracted. Based on the comparison matrix, a series of calculations are performed to choose the best altemative

Applying the AHP method, the overall performance could be calculated. There are 2 sets of data, the first set is that equal ratings are assigned to all 4 parameters. The second set is that the overall performance emphases on average order lead time, i.e. 0.4 rating is assigned to the average order lead time, and 0.2 rating is assigned to remaining three parameters. As a result, the higher overall value in the AHP analysis, the better performance is the supply chain model.

The results are shown in Table 2 , the best performance under equal rating is Model 3 (Network Supply Chain). Communication between Manufacturer layers and for the unfulfilled order equally distributed to Suppliers were the best approach. From the simulation results, the performance of the four parameters is quite satisfied.

The results associated to set 2 experiment (Table 3 ), the best performance under time-focused rating is still Model 3 (Network Supply Chain). Using the AHP method, by setting the different rating in the para meters, different performance results would be obtained.

\section{Conclusions}

In this paper, the principle of the co-operation just applied in suppliers and manufacturers. In fact, it could extend in downstream distributors, retailers and even end users. The new competitive paradigm is that supply chain competes with supply chain and the success of any one company will depend upon how well it manages its supply chain relationships. Simulation results proved that the Network supply chain model is better than he traditional one namely Inter-organizational model. From the data of the simulation, although the Network supply chain model is better than the regional clustering supply chain, regional clustering supply chain models could be potentially developed to the most optimal one.

\section{References}

[1] T. L. Satty, The Analytic Hierarchy Process: Planning, Priority Setting, Resource Allocation. RWS Publication, 1990.

[2] C. Harland, "Supply network strategy: observations on structure, infrastructure and operations performance", Proceedings of the $2^{\text {od }}$ Worldwide Symposium on Purchasing and Supply Chain Management, IPSERA, (London, U.K.), pp. 248-267, 1998.

[3] J. Jarillo, “On strategic networks", Strategic Management Journal, no.9, pp. 31-41, 1998.

[4] R. Hall, R. "Developing inter-organisational strategy", Proceedings of the $2^{\text {nd }}$ Worldwide Symposium on Purchasing and Supply Chain Management, IPSERA, (London,U.K.), pp. 229-47, 1998.

[5] A. Esain, "Regional innovation through intercompany networking: evidence from Wales", Proceedings of the $3^{d}$ International Symposium on Logistics, (The University of Padua), pp. 87-96, 1997. 


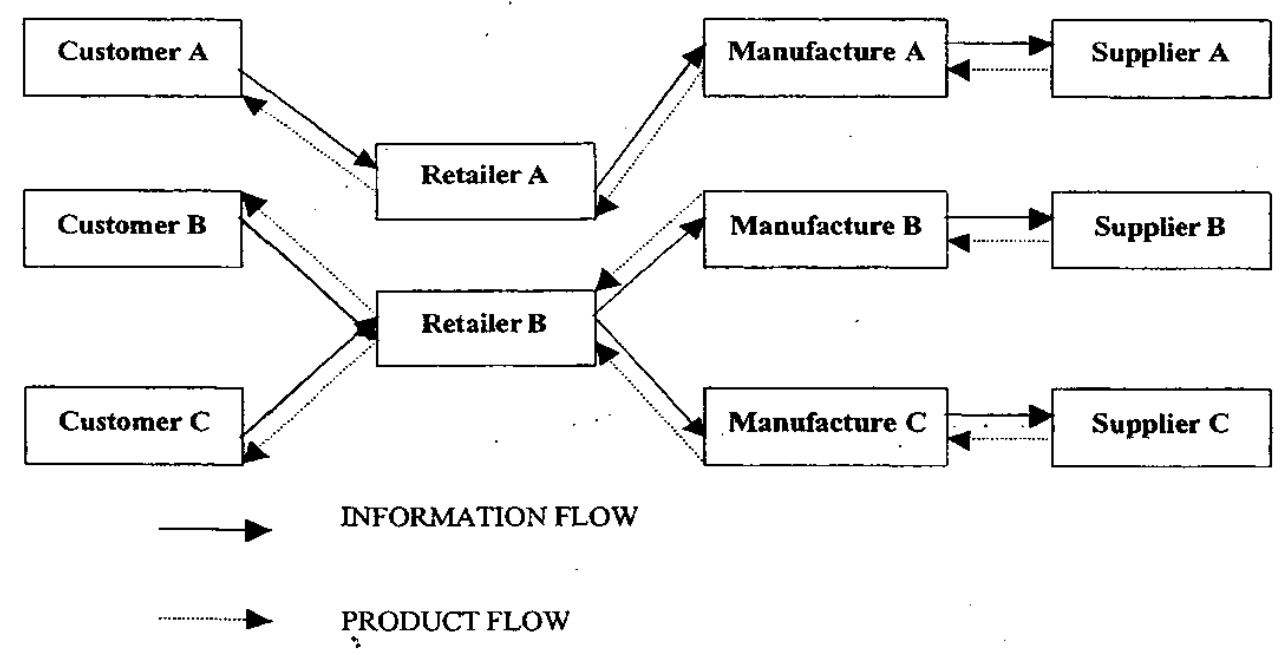

Figure 1. Inter-organizational Supply Chain Model (Model 1)

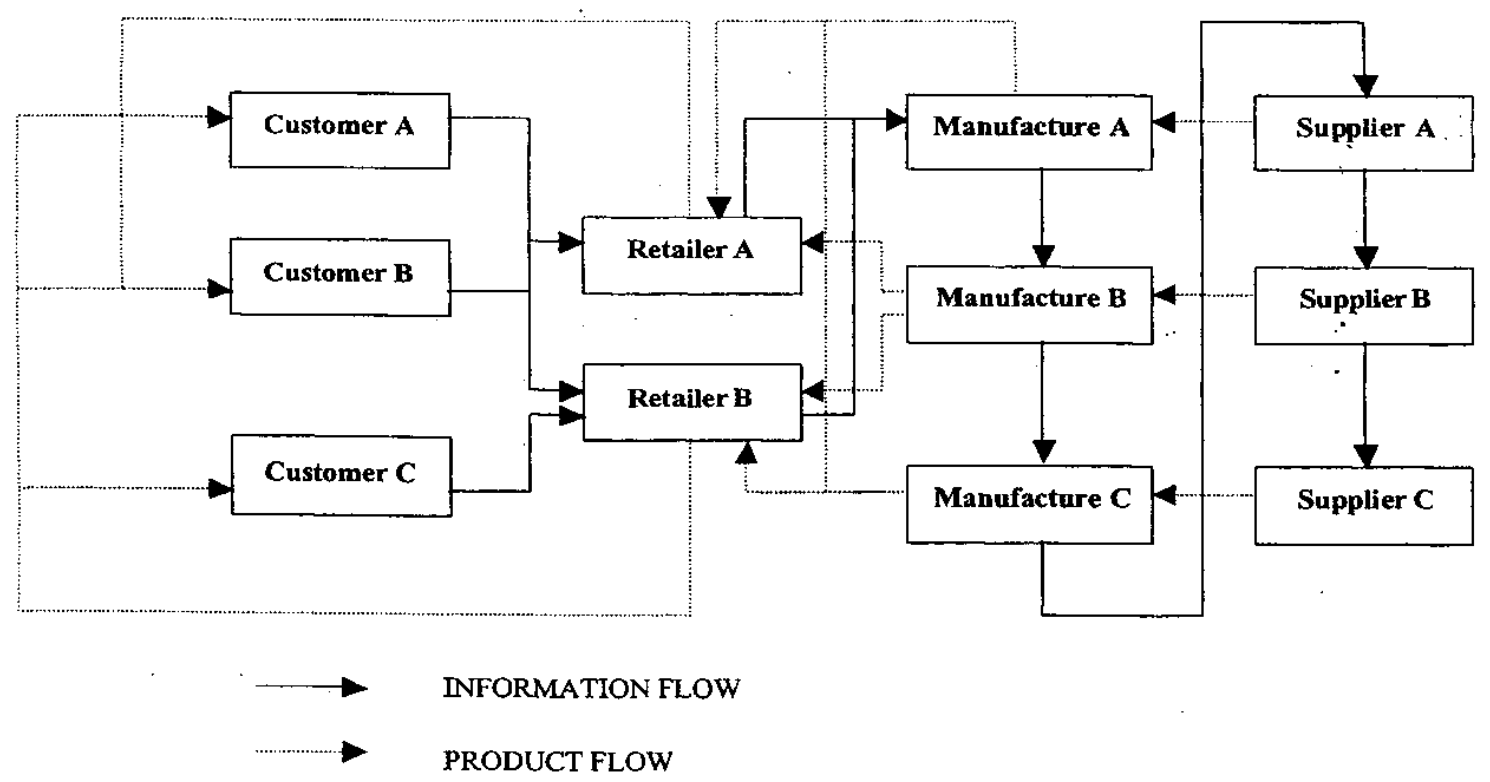

Figure 2. Network Supply Chain Model (Models 2-4) 


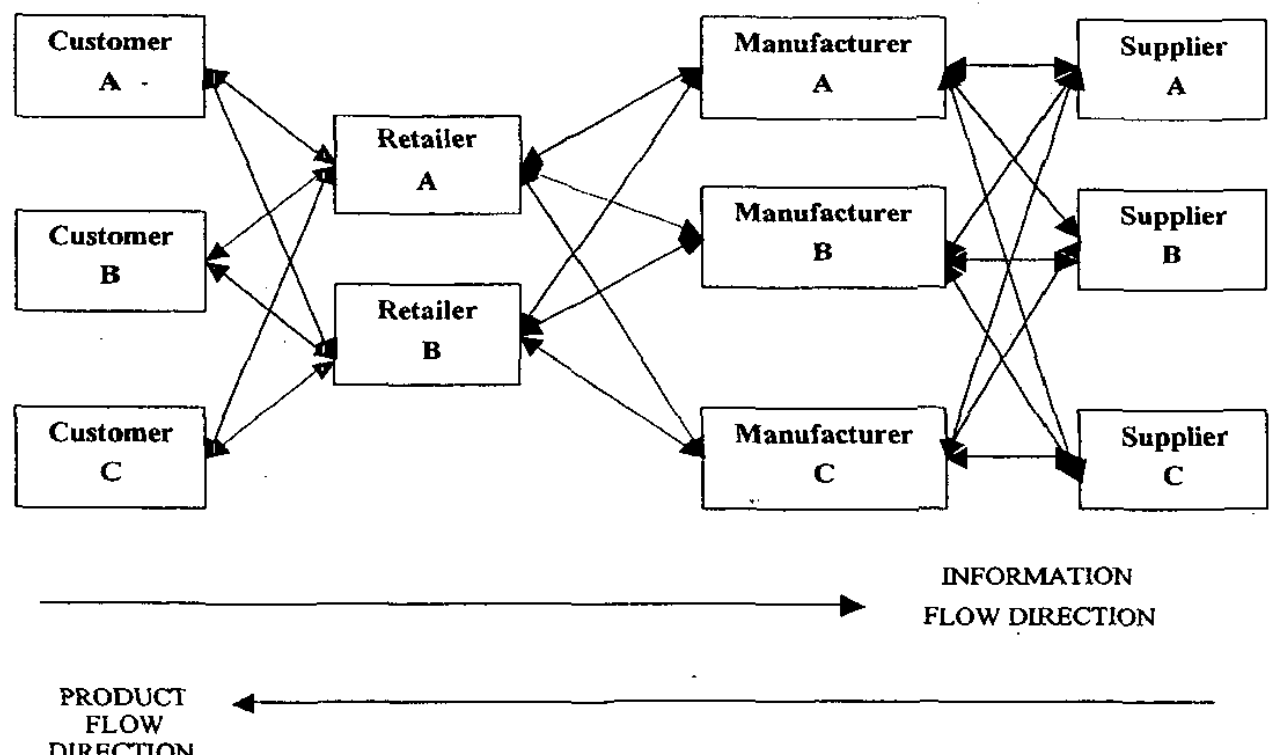

Figure 3. Regional clustering Supply Chains Model (Model 5)

Table 1. Overall performance

\begin{tabular}{|c|c|c|c|c|}
\hline Model No. & $\begin{array}{c}\text { Average Order } \\
\text { Lead Time (hours) }\end{array}$ & $\begin{array}{c}\text { Resources } \\
\text { Utilization } \\
(\%)\end{array}$ & $\begin{array}{c}\text { Average Inventory } \\
\text { Level } \\
\text { (units) }\end{array}$ & $\begin{array}{c}\text { Transportation } \\
\text { Cost (\$) }\end{array}$ \\
\hline 1 & 34 & 10.56 & 1750 & 4235 \\
\hline 2 & 25.5 & 16.05 & 1736 & 4832 \\
\hline 3 & 28 & 20.6 & 700 & 5058 \\
\hline 4 & 28.9 & 20.89 & 790 & 5822 \\
\hline 5 & 37 & 44.87 & 0 & 5708 \\
\hline
\end{tabular}

Table 2. AHP result with Set 1-Equal Rating

\begin{tabular}{|c|c|c|c|c|c|}
\hline & Model 1 & Model 2 & Model 3 & Model 4 & Model 5 \\
\hline Rating & 0.5 & 0.5 & 0.65 & 0.55 & 0.6 \\
\hline
\end{tabular}

Table 3. AHP result with Set 2-Time Based Rating

\begin{tabular}{|cc|c|c|c|c|}
\hline & Model 1 & Model 2 & Model 3 & Model 4 & Model 5 \\
\hline Rating & $\mathbf{0 . 4 8}$ & 0.52 & 0.6 & 0.56 & 0.52 \\
\hline
\end{tabular}

